REVISTA DE LA ESCUELA DE CIENCIAS DE LA EDUCACIÓN, AÑO 16, NRO. 15, VOL. 2, JULIO A DICIEMRE DE 2020. PÁGINAS 45-57. ISSN 2362-3349 (EN LÍNEA). EL PUZLE COMO TÉCNICA DE APRENDIZAJE COOPERATIVO PARA LA ENSEÑANZA DE LA HISTORIA Y EL DESARROLLO DE HABILIDADES BLANDAS. HUMBERTO ANDRÉS ÁLVAREZ SEPÚLVEDA.

\title{
EL PUZLE COMO TÉCNICA DE APRENDIZAJE COOPERATIVO PARA LA ENSEÑANZA DE LA HISTORIA Y EL DESARROLLO DE HABILIDADES BLANDAS
}

\author{
Humberto Andrés Álvarez Sepúlveda* \\ Facultad de Educación de la Universidad Católica de la Santísima Concepción, Chile \\ halvarez@ucsc.cl
}

Recibido: 22/09/2019 Aceptado: 23/10/2019

\begin{abstract}
Resumen
Dentro de la enseñanza universitaria de la Historia, el aprendizaje cooperativo se presenta como una metodología en la que el alumnado puede desarrollar su pensamiento histórico y potenciar sus habilidades blandas. Entre las técnicas más idóneas para lograr dichos fines, destaca el puzle creado por Elliot Aronson en 1971. El presente artículo tiene por objetivo describir la implementación de esta técnica en un grupo de estudiantes de Pedagogía de la Universidad Católica de la Santísima Concepción (Chile). La metodología usada es cualitativa y se basa en un estudio descriptivo de caso. Los resultados obtenidos de la experiencia demuestran que el puzle es altamente efectivo para generar un aprendizaje significativo en los estudiantes, pues, según la perspectiva docente y la de ellos mismos, lograron perfeccionar sus habilidades blandas (trabajo colaborativo, comunicación oral, compañerismo, responsabilidad individual y grupal, entre otras) y mejorar su comprensión sobre la Historia y su didáctica.
\end{abstract}

Palabras clave: Enseñanza universitaria - Historia - Aprendizaje cooperativo - Puzle - Pensamiento histórico.

\begin{abstract}
Within the university teaching of history, cooperative learning is presented as a methodology in which students can develop their historical thinking and enhance their soft skills. The puzzle created by Elliot Aronson in 1971 stands out among the most suitable techniques to achieve these ends. The objective of this article is to describe the implementation of this technique in a group of students of Pedagogy at the Catholic University of the Most Holy Conception (Chile). The methodology used is qualitative and is based on a descriptive case study. The results obtained from the experience show that the puzzle is highly effective in generating significant learning in the students, since, according to the teaching perspective and that of the students themselves, they were able to perfect their soft skills (collaborative work, oral communication, companionship, individual and group responsibility, among others) and improve their understanding of History and its didactics.
\end{abstract}

Keywords: University teaching - History - Cooperative learning - Puzzle - Historical thinking.

*Doctor en Sociedad y Cultura y Máster en Historia Contemporánea y Mundo Actual por la Universidad de Barcelona (España). Profesor en Historia y Geografía, Licenciado en Educación y Licenciado en Historia por la Universidad Católica de la Santísima Concepción. Investigador en la formación de profesores de Historia, la enseñanza, el aprendizaje y la evaluación de la especialidad. Académico de la Facultad de Educación de la Universidad Católica de la Santísima Concepción. 
REVISTA DE LA ESCUELA DE CIENCIAS DE LA EDUCACIÓN, AÑO 16, NRO. 15, VOL. 2, JULIO A DICIEMRE DE 2020. PÁGINAS 45-57. ISSN 2362-3349 (EN LÍNEA). EL PUZLE COMO TÉCNICA DE APRENDIZAJE COOPERATIVO PARA LA ENSEÑANZA DE LA HISTORIA Y EL DESARROLLO DE HABILIDADES BLANDAS. HUMBERTO ANDRÉS ÁLVAREZ SEPÚLVEDA.

\section{Introducción}

En el siglo XXI, el enfoque basado en resultados de aprendizaje y competencia ha tenido un importante auge debido a que la política educativa actual y la ideología de mercado subrayan las ventajas que tienen las competencias como referente de la educación para el trabajo y la formación profesional (Biberhofer et al., 2018; García, 2019; Rodríguez et al., 2019; Rumbo y Gómez, 2019).

Según García (2019), la competencia se entiende como una combinación dinámica de conocimientos, habilidades, actitudes y responsabilidades que describen los resultados de aprendizaje de un programa educativo. Esto es, la capacidad de una persona para enfrentarse con garantías de éxito a una tarea en un contexto determinado.

Siguiendo dicho enfoque, el sistema educativo tiene como principal objetivo la satisfacción de necesidades de aprendizaje de las personas; en este marco, la universidad, a partir del enfoque por competencias, tiene la misión de superar los modelos pedagógicos de transmisión y de formar en los saberes pertinentes para enfrentar las nuevas demandas del mercado laboral.

En el ámbito de la enseñanza universitaria de la Historia, y con el fin de adaptarse al enfoque por competencias, es necesario transitar desde un paradigma tradicional a un modelo constructivista donde el profesor sea el mediador de un aprendizaje significativo para sus alumnos (Gómez et al., 2018; Miralles y Monteagudo, 2019).

Para concretar este avance, es fundamental que los profesores universitarios estén dispuestos a implementar metodologías de aprendizaje cooperativo, las cuales, según Pinos (2019), comprenden todos aquellos métodos, técnicas y estrategias que utiliza el docente para convertir el proceso de enseñanza en actividades que fomenten el trabajo en equipo y la participación autónoma del estudiante. Esto implica modificar la planificación de las asignaturas, el desarrollo de las actividades formativas y la evaluación de los aprendizajes, de tal manera que se promueva un alineamiento constructivista en la práctica docente.

Dentro de las técnicas más utilizadas para desarrollar el aprendizaje cooperativo, destaca el puzle, también conocido como rompecabezas, puesto que es una de las opciones didácticas más eficaces para motivar el gusto por la Historia, reducir las ausencias y mejorar el desempeño académico (González y Núñez, 2012; Salazar et al., 2017).

El presente artículo tiene por objetivo describir una experiencia de innovación basada en la implementación de la técnica del puzle en un grupo de estudiantes de Pedagogía de la Universidad Católica de la Santísima Concepción (Chile), con el fin de evaluar su eficacia en el desarrollo de habilidades blandas y de aprendizajes significativos relacionados con la Historia y su didáctica. Esto surge de la necesidad de contrarrestar la pasividad del alumnado en su proceso de aprendizaje.

Dicha necesidad surge a raíz de una reflexión personal realizada sobre la propia práctica docente. Si bien la mayoría de las veces se diseñan una serie de actividades y propuestas metodológicas con la noble intención de promover oportunidades de aprendizaje, en la praxis se tiende a caer de forma recurrente en la clase magistral que no permite a los estudiantes potenciar sus habilidades sociales o mejorar su comprensión en la asignatura impartida. Esta situación motivó a reformular la enseñanza partiendo de la siguiente premisa: el desarrollo de habilidades blandas y la calidad de los aprendizajes relacionados con la Historia y su didáctica mejorarán considerablemente con la implementación de la experiencia basada en el puzle.

\section{El puzle en la enseñanza universitaria de la Historia}

La incorporación de técnicas de aprendizaje cooperativo en la enseñanza universitaria de la Historia es uno de los grandes desafíos de los sistemas educativos actuales, pues todavía se encuentran anclados en un tradicionalismo academicista caracterizado por el predominio de la clase magistral y de evaluaciones focalizadas mayormente en la repetición y memorización de contenidos (Gómez et al., 2018; Miralles y Monteagudo, 2019).

Este modelo de enseñanza contribuye a agudizar la pereza que tienen muchos docentes por innovar con metodologías activas (Rivera y Mondaca, 2013) y a radicalizar la percepción general del alumnado que concibe la Historia como una materia fácil, aburrida y poco útil, y que tan solo exige buena memoria (Barton, 2010; Barca, 2011; Prats, 2017).

A raíz de esta situación, y de la propia reflexión personal realizada, resulta indispensable reorientar la enseñanza de la Historia mediante la implementación de experiencias didácticas basadas en estrategias innovadoras como el rompecabezas, que presenta un gran potencial para desarrollar el pensamiento histórico y mejorar las habilidades blandas (González y Núñez, 2012; Salazar et al., 2017).

El puzle, diseñado por Elliot Aronson en 1971 (Aronson y Blaney, 1978), consiste en una técnica de aprendizaje cooperativo que inicia con la conformación de grupos base reducidos y la distribución del material docente en partes equitativas entre los integrantes de los equipos; en segundo lugar, prosigue con el estudio autónomo de la parte asignada; en tercer lugar, continua con la organización de los comités de expertos para profundizar en la temática: en cuarto lugar, sigue con la constitución de los grupos base colaborativos para 
REVISTA DE LA ESCUELA DE CIENCIAS DE LA EDUCACIÓN, AÑO 16, NRO. 15, VOL. 2, JULIO A DICIEMRE DE 2020. PÁGINAS 45-57. ISSN 2362-3349 (EN LÍNEA). EL PUZLE COMO TÉCNICA DE APRENDIZAJE COOPERATIVO PARA LA ENSEÑANZA DE LA HISTORIA Y EL DESARROLLO DE HABILIDADES BLANDAS. HUMBERTO ANDRÉS ÁLVAREZ SEPÚLVEDA.

que cada miembro, por turnos, enseñe su parte al resto de los integrantes; y, por último, finaliza con reflexión grupal de cada equipo.

Debido a su innovadora metodología de trabajo, esta técnica contribuye a organizar y conducir la enseñanza en el aula, potencia la participación del estudiante y favorece la actividad de estilos de aprendizaje diferentes (Gómez et al., 2017). Adicionalmente, ayuda a disminuir la conflictividad en el aula, fomenta la interdependencia positiva entre los estudiantes, reduce el absentismo y mejora la autoestima, especialmente de los alumnos con bajo rendimiento académico (González y Núñez, 2012; Salazar et al., 2017).

Para lograr estos propósitos, el docente debe satisfacer, al momento de implementar el puzle, los siguientes cinco aspectos del aprendizaje cooperativo (Johnson et al., 1999; Pinos, 2019):

1. Interdependencia positiva: Una tarea de grupo tiene interdependencia positiva cuando todos los miembros del mismo son necesarios e indispensables para que la tarea pueda realizarse con éxito. Es decir, no es posible que uno de los integrantes del equipo (o un subgrupo) realice la tarea por su cuenta, al margen de los demás.

2. Exigibilidad individual: Una tarea tiene exigibilidad individual cuando cada uno de los integrantes del equipo debe rendir cuentas no sólo de su parte del trabajo, sino también del realizado por el resto del grupo. De ahí la relevancia que asume la responsabilidad individual y grupal en el contexto del aprendizaje cooperativo.

3. Interacción cara a cara: Aunque hoy en día las herramientas telemáticas permiten el trabajo grupal a distancia, es importante tener en cuenta que el desempeño mejora cuando el equipo interactúa físicamente con una cierta periodicidad. De hecho, algunas habilidades importantes del trabajo colaborativo (por ejemplo, emitir gestos de apoyo a las propuestas de los demás) sólo pueden ponerse en práctica cuando los integrantes de un equipo se comunican cara a cara.

4. Habilidades interpersonales y de trabajo en grupo: Trabajar en equipo de forma eficaz no es fácil, puesto que son frecuentes los casos de grupos o individuos que son incapaces de llegar a un acuerdo, de formular críticas constructivas, o de aceptarlas sin sentirse ofendidos. En estas situaciones, el profesor debe estar preparado para ayudar a sus estudiantes a resolver los conflictos de una forma respetuosa y consensuada.

5. Reflexión del grupo: Para facilitar el desarrollo de habilidades blandas, los equipos deben someterse de vez en cuando a actividades de reflexión, en las que puedan identificarse las ventajas y dificultades del trabajo colaborativo. Algunas de estas actividades de reflexión deben ser programadas por el profesor, aunque idealmente los grupos deben aprender a identificar cuándo deben sentarse a reflexionar y cómo organizar dicha instancia. También para esta dimensión existen innumerables ejemplos de cuestionarios y materiales que facilitan el proceso de reflexión y ayudan a explicitar la naturaleza de las dificultades del grupo.

Con el objetivo de velar por el cumplimiento de cada uno de los aspectos concernientes al aprendizaje cooperativo, es indispensable concebir el proceso formativo como una docencia al servicio del estudiante. Por tal motivo, bajo este paradigma, el profesor asume un rol de guía y mediador que permite enfocar las disposiciones del trabajo colaborativo, a través de actividades de aprendizaje que estimulan en el estudiante la participación, cooperación, creatividad y reflexión sobre la tarea solicitada (Salazar et. al. 2017). Por otra parte, cabe destacar que el puzle en el ámbito de la enseñabilidad de la Historia se concibe como una técnica de indagación, que tiene como objetivo poner al alumnado en situaciones de reflexión y acción mediante el desarrollo de conceptos, procedimientos y actitudes (Gómez et. al. 2017). En esta línea, esta estrategia rompe con la línea de la enseñanza tradicional, pues plantea una reforma del pensamiento y una manera distinta de construir el conocimiento histórico.

Gracias a dicha cualidad, el puzle puede desarrollar el pensamiento histórico en los alumnos, pues les ayuda a indagar con mayor profundidad en los antecedentes históricos del eje temático asignado y a contrastar las diversas interpretaciones historiográficas investigadas (Salazar et. al. 2017). Por tal motivo, el planteamiento didáctico de los contenidos históricos tiene que incorporar situaciones o problemas de interés para el alumnado o bien temáticas donde se ponga de manifiesto la necesidad de discutir o de establecer nuevas miradas.

Para promover el desarrollo del pensamiento histórico en el alumnado, es imprescindible que el docente sea capaz de convertir una parte de las actividades didácticas en un proceso de aprendizaje por descubrimiento, lo que facilita la constitución de la clase como un laboratorio de historia (Prats, 2011; Gómez et. al. 2017). Esta instancia puede incorporarse en el rompecabezas para introducir a los estudiantes en el trabajo historiográfico y así puedan analizar, bajo la guía del profesor, diversas fuentes de información para 
REVISTA DE LA ESCUELA DE CIENCIAS DE LA EDUCACIÓN, AÑO 16, NRO. 15, VOL. 2, JULIO A DICIEMRE DE 2020. PÁGINAS 45-57. ISSN 2362-3349 (EN LÍNEA). EL PUZLE COMO TÉCNICA DE APRENDIZAJE COOPERATIVO PARA LA ENSEÑANZA DE LA HISTORIA Y EL DESARROLLO DE HABILIDADES BLANDAS. HUMBERTO ANDRÉS ÁLVAREZ SEPÚLVEDA.

que, de forma autónoma, puedan profundizar en su parte asignada, conformar comités de expertos y establecer los grupos base colaborativos.

Como señalan Salazar, Orellana, Muñoz y Bellati (2017), el laboratorio histórico dentro de la técnica del puzle busca que los alumnos apliquen cuatro heurísticas durante la lectura de textos históricos:

1. Heurística de origen, teniendo en cuenta de donde proviene el documento histórico y su propósito.

2. Contextualización, con el fin de colocar el documento en su contexto temporal y espacial.

3. Lectura cerrada, para extraer la principal idea del documento.

4. Corroboración heurística, para comparar las múltiples fuentes.

A partir de los procedimientos mencionados, el alumnado puede pensar históricamente, es decir, comenzar con la indagación disciplinada que lo motive a responder preguntas históricas, encontrar información, evaluar fuentes y conciliar e interpretar diversas explicaciones (Levstik y Barton, 2011; Boutonnet, 2013; Sáiz y Domínguez, 2017; Miralles y Monteagudo, 2019). Para ello, es fundamental que el docente pueda promover en los estudiantes aprendizajes que vayan más allá de conocimientos declarativos de primer orden (el qué, dónde, cuándo o quién ha llevado a cabo las distintas acciones bajo un relato secuencial), puesto que el enfoque del pensamiento histórico está dirigido a privilegiar procedimientos y aprendizajes de segundo orden (tabla 1).

Tabla 1. Aprendizajes de segundo orden del pensamiento histórico

\begin{tabular}{|l|l|}
\hline Concepto & Definición \\
\hline Evidencias históricas & $\begin{array}{l}\text { Este procedimiento permite ir de un análisis descriptivo de las fuentes a un } \\
\text { análisis heurístico y contextualizado. }\end{array}$ \\
\hline $\begin{array}{l}\text { Tiempo histórico, cambio y } \\
\text { continuidad }\end{array}$ & $\begin{array}{l}\text { Este concepto implica comprender conceptos y convenciones temporales y } \\
\text { cronológicas para situar los procesos de cambio y continuidad en la dimensión } \\
\text { pasado-presente y futuro de la historia. }\end{array}$ \\
\hline Relevancia histórica & $\begin{array}{l}\text { Este concepto busca apreciar la importancia de fenómenos del pasado en el } \\
\text { presente y valorar el significado cambiante de la relevancia según los } \\
\text { intereses de actores sociales actuales. }\end{array}$ \\
\hline $\begin{array}{l}\text { Empatía histórica } \\
\text { consecuencias }\end{array}$ & $\begin{array}{l}\text { Este concepto pretende comprender la historicidad y lejanía del pasado para } \\
\text { evitar juzgar los hechos históricos desde la óptica presentista. }\end{array}$ \\
\hline Dimensión ética & $\begin{array}{l}\text { Este concepto permite comprender la multicausalidad de los fenómenos del } \\
\text { pasado e identificar y clasificar las causas de los fenómenos de forma } \\
\text { descriptiva y explicativa. }\end{array}$ \\
\hline $\begin{array}{l}\text { Relatos o interpretaciones } \\
\text { historiográficas }\end{array}$ & $\begin{array}{l}\text { Este concepto implica identificar y comprender juicios éticos sobre fenómenos } \\
\text { del pasado de forma contextualizada. }\end{array}$ \\
\hline Uso de la historia & $\begin{array}{l}\text { Este concepto busca asumir la existencia de distintas interpretaciones } \\
\text { historiográficas como un rasgo distintivo del conocimiento histórico. }\end{array}$ \\
\hline & $\begin{array}{l}\text { Este concepto pretende comprender la utilización y presencia social y pública } \\
\text { del pasado para explicar o justificar hechos del presente, conformar } \\
\text { identidades e influir en el futuro. }\end{array}$ \\
\hline
\end{tabular}

Fuente: Versión adaptada de Sáiz y Domínguez (2017)

\section{Metodología}

La investigación es cualitativa y se basa en el estudio descriptivo de caso (Simons, 2011; Díaz y Luna, 2014). Su objetivo, como ya se mencionó, consiste en analizar la implementación de la técnica del puzle en un grupo de estudiantes de Pedagogía de la Universidad Católica de la Santísima Concepción (Chile). El método de recolección de datos fue la observación participante de las clases donde se aplicó la experiencia.

Adicionalmente, el estudio es una investigación evaluativa (Escudero, 2016), que tiene como propósito recabar información sistemática sobre la experiencia para desarrollar una evaluación formativa del proceso de enseñanza-aprendizaje y, a partir de ella, tomar decisiones de mejora que beneficien a los estudiantes.

La investigación se enmarca en el paradigma crítico (Melero, 2012), cuyos lineamientos se dirigen hacia la emancipación social y la democratización del currículum. Desde esta perspectiva, este estudio pretende contribuir a contrarrestar el predominio de la enseñanza tradicional de la Historia, evidenciando la eficacia de las técnicas de aprendizaje cooperativo, en este caso del rompecabezas, para promover aprendizajes significativos relacionados con dicha especialidad. 
REVISTA DE LA ESCUELA DE CIENCIAS DE LA EDUCACIÓN, AÑO 16, NRO. 15, VOL. 2, JULIO A DICIEMRE DE 2020. PÁGINAS 45-57. ISSN 2362-3349 (EN LÍNEA). EL PUZLE COMO TÉCNICA DE APRENDIZAJE COOPERATIVO PARA LA ENSEÑANZA DE LA HISTORIA Y EL DESARROLLO DE HABILIDADES BLANDAS.

HUMBERTO ANDRÉS ÁLVAREZ SEPÚLVEDA.

\section{Participantes}

Como se constata en la tabla 2 , los sujetos participantes $(p=36)$ son estudiantes de las asignaturas "Didáctica de la Historia, Geografía y Ciencias Sociales I" ( $p=12)$, "Ciencias Sociales II" $(p=8)$ y "Conociendo Chile de los siglos XIX y XX" ( $p=16)$. La muestra se compone de 26 mujeres $(72 \%)$ y 10 hombres $(28 \%)$.

Tabla 2. Datos cuantitativos de los sujetos participantes según asignaturas incluidas en el puzle

\begin{tabular}{|l|l|l|l|l|l|l|}
\hline $\begin{array}{l}\text { Nombre de la } \\
\text { actividad } \\
\text { curricular }\end{array}$ & Carrera & $\begin{array}{l}\text { Semestre de } \\
\text { la Carrera }\end{array}$ & Semestre/año & $\begin{array}{l}\text { Cantidad } \\
\text { de alumnos }\end{array}$ & Hombres & Mujeres \\
\hline $\begin{array}{l}\text { Didáctica de la } \\
\text { Historia, } \\
\text { Geografía y } \\
\text { Ciencias } \\
\text { Sociales I }\end{array}$ & $\begin{array}{l}\text { Programa de } \\
\text { Formación } \\
\text { Pedagógica }\end{array}$ & $\begin{array}{l}\text { Primer } \\
\text { semestre }\end{array}$ & $\begin{array}{l}\text { Primer } \\
\text { semestre/2019 }\end{array}$ & 12 & 7 & 5 \\
\hline $\begin{array}{l}\text { Ciencias } \\
\text { Sociales II }\end{array}$ & $\begin{array}{l}\text { Pedagogía en } \\
\text { Educación } \\
\text { General Básica } \\
\text { con Menciones }\end{array}$ & $\begin{array}{l}\text { Quinto } \\
\text { semestre }\end{array}$ & $\begin{array}{l}\text { Primer } \\
\text { semestre/2019 }\end{array}$ & 8 & & \\
\hline $\begin{array}{l}\text { Conociendo } \\
\text { Chile de los } \\
\text { siglos XIX y } \\
\text { XX }\end{array}$ & $\begin{array}{l}\text { Pedagogía en } \\
\text { Educación } \\
\text { Ceneral Básica }\end{array}$ & $\begin{array}{l}\text { Séptimo } \\
\text { semestre }\end{array}$ & $\begin{array}{l}\text { Primer } \\
\text { semestre/2019 }\end{array}$ & 16 & 1 & 7 \\
\hline
\end{tabular}

Fuente: Elaboración propia

La técnica de muestreo aplicada fue de carácter no probabilístico por conveniencia (Gómez, 2006) y no estratificado (Hernández et. al. 2014), puesto que el estudio requirió de estudiantes que hayan cursado asignaturas donde se impartan contenidos vinculados a la Historia y su didáctica. La selección total de la muestra dependió del investigador, y no de una probabilidad aleatoria de elección, respondiendo a criterios selectivos intencionales y a las posibilidades de acceso a la misma, en función del grado de adecuación a los objetivos de estudio.

\section{Contexto de aplicación}

Las asignaturas "Didáctica de la Historia, Geografía y Ciencias Sociales I", "Ciencias Sociales II" y "Conociendo Chile de los siglos XIX y XX" son impartidas a través del Departamento de Didáctica de la Universidad Católica de la Santísima Concepción (Chile).

El carácter de estas actividades curriculares es teórico-práctico, el cual se traduce en el desarrollo de clases centradas en la discusión socializada y en la implementación de talleres basados en el uso de metodologías activas, que tienen como propósito desarrollar el pensamiento histórico en la formación inicial docente.

En las asignaturas mencionadas, y a efectos de esta investigación, el rompecabezas se presenta como una técnica didáctica que busca mejorar la calidad de los aprendizajes en el marco de las siguientes unidades y temáticas:

Tabla 3. Consignación de temáticas por asignatura

\begin{tabular}{|c|c|c|c|}
\hline Asignatura & Unidad & Temática & $\begin{array}{l}\text { Partes distribuidas entre los } \\
\text { integrantes del equipo de trabajo }\end{array}$ \\
\hline $\begin{array}{l}\text { Didáctica de la } \\
\text { Historia, } \\
\text { Geografía y } \\
\text { Ciencias } \\
\text { Sociales I }\end{array}$ & $\begin{array}{l}\text { Unidad I: "La Didáctica } \\
\text { de la Especialidad y su } \\
\text { objeto de estudio". }\end{array}$ & $\begin{array}{l}\text { Por qué y para qué } \\
\text { enseñar Historia (Primer } \\
\text { capítulo del libro "Didáctica } \\
\text { de la Geografía y la } \\
\text { Historia", coordinado por } \\
\text { Joaquim Prats). }\end{array}$ & $\begin{array}{l}\text { Parte 1: El valor formativo de la } \\
\text { Historia. } \\
\text { Parte 2: Las funciones de la } \\
\text { historia en nuestra sociedad. } \\
\text { Parte 3: Usos y abusos de la } \\
\text { enseñanza de Historia. } \\
\text { Parte 4: Los fines generales de la } \\
\text { Historia como materia educativa. }\end{array}$ \\
\hline $\begin{array}{l}\text { Ciencias } \\
\text { Sociales II }\end{array}$ & $\begin{array}{l}\text { Unidad I: "La llegada de } \\
\text { europeos a la bautizada } \\
\text { América". }\end{array}$ & $\begin{array}{l}\text { El perfil del conquistador y } \\
\text { la expedición de Diego de } \\
\text { Almagro y de Pedro de } \\
\text { Valdivia (Texto preparado } \\
\text { por el docente). }\end{array}$ & $\begin{array}{l}\text { Parte 1: El perfil del } \\
\text { conquistador. } \\
\text { Parte 2: La organización de la } \\
\text { empresa de conquista. } \\
\text { Parte 3: La expedición de Diego }\end{array}$ \\
\hline
\end{tabular}


REVISTA DE LA ESCUELA DE CIENCIAS DE LA EDUCACIÓN, AÑO 16, NRO. 15, VOL. 2, JULIO A DICIEMRE DE 2020. PÁGINAS 45-57. ISSN 2362-3349 (EN LÍNEA). EL PUZLE COMO TÉCNICA DE APRENDIZAJE COOPERATIVO PARA LA ENSEÑANZA DE LA HISTORIA Y EL DESARROLLO DE HABILIDADES BLANDAS.

\begin{tabular}{|l|l|l|l|}
\hline & & & $\begin{array}{l}\text { de Almagro. } \\
\text { Parte 4: La expedición de Pedro } \\
\text { de Valdivia. }\end{array}$ \\
\hline $\begin{array}{l}\text { Conociendo } \\
\text { shigles XIX y XX } \\
\text { dependencias". }\end{array}$ & $\begin{array}{l}\text { Unidad I: "Emancipación } \\
\text { de España y nuevas }\end{array}$ & $\begin{array}{l}\text { Los antecedentes de la } \\
\text { Independencia de Chile } \\
(1810-1823) . \quad \begin{array}{l}\text { Parte 1: La invasión de Napoleón } \\
\text { internos y externos (Texto } \\
\text { preparado por el docente). }\end{array} \\
\text { a España y el cautiverio del Rey } \\
\text { Fernando VII. } \\
\text { Parte 2: La ilustración y la } \\
\text { Independencia de los Estados } \\
\text { Unidos. } \\
\text { Parte 3: Crisis del monopolio } \\
\text { comercial español en América. } \\
\text { Parte 4: El descontento criollo. }\end{array}$ \\
\hline
\end{tabular}

Fuente: Elaboración propia

Siguiendo la distribución de la tabla 3, los estudiantes organizados en equipos de cuatro integrantes tuvieron que preparar y analizar la temática asignada.

Los aspectos considerados para trabajar en el taller tienen relación con el conocimiento histórico de los temas consignados para cada asignatura (tabla 3) y con contenidos vinculados a la Didáctica de la Historia. Estos últimos fueron comunes y transversales para todas las actividades curriculares y apuntan principalmente a las competencias cognitivas del pensamiento histórico y a la metodología de trabajo del puzle.

En el transcurso de la experiencia, los estudiantes participaron durante tres sesiones en las siguientes fases de trabajo:

Fase I. Preparación de la experiencia y conformación de los grupos base: En las dos semanas previas al puzle, el docente explica todos los contenidos necesarios y especificados para cada curso, haciendo especial mención a la metodología y al propósito de la actividad. Esta explicación es complementada con una guía de trabajo que describe las fases operativas del rompecabezas. Luego, se conforman equipos de cuatro integrantes, quedando organizados por afinidad de la siguiente manera:

Tabla 4. Conformación de los equipos de trabajo según curso

\begin{tabular}{|l|l|l|}
\hline Asignatura & Grupo & Estudiantes \\
\hline Didáctica de la Historia, Geografía y Ciencias Sociales I & Grupo 1 & P1+P2+P3+P4 \\
\hline Didáctica de la Historia, Geografía y Ciencias Sociales I & Grupo 2 & P5+P6+P7+P8 \\
\hline Didáctica de la Historia, Geografía y Ciencias Sociales I & Grupo 3 & P9+P10+P11+P12 \\
\hline Ciencias Sociales II & Grupo 4 & P17+P18+P19+P20 \\
\hline Ciencias Sociales II & Grupo 5 & P21+P22+P23+P24 \\
\hline Conociendo Chile de los siglos XIX y XX & Grupo 6 & P25+P26+P27+P28 \\
\hline Conociendo Chile de los siglos XIX y XX & Grupo 7 & P29+P30+P31+P32 \\
\hline Conociendo Chile de los siglos XIX y XX & Grupo 8 & P33+P34+P35+P36 \\
\hline Conociendo Chile de los siglos XIX y XX & Grupo 9 & \\
\hline
\end{tabular}

Fuente: Elaboración propia

Fase II. Introducción al puzle: En la sesión 1, el docente entrega el texto de estudio a los grupos y lo divide en 4 temas para distribuirlo, en la próxima fase, entre todos los integrantes. El profesor elige al azar a un líder de cada equipo. Esta fase toma alrededor de 5 minutos. 
REVISTA DE LA ESCUELA DE CIENCIAS DE LA EDUCACIÓN, AÑo 16, NRO. 15, VOL. 2, JULIO A DICIEMRE DE 2020. PÁGINAS 45-57. ISSN 2362-3349 (EN LÍNEA). EL PUZLE COMO TÉCNICA DE APRENDIZAJE COOPERATIVO PARA LA ENSEÑANZA DE LA HISTORIA Y EL DESARROLLO DE HABILIDADES BLANDAS. HUMBERTO ANDRÉS ÁLVAREZ SEPÚLVEDA.

Fase III. Trabajo autónomo: El docente le asigna un número al azar (del 1 al 4) a cada integrante del grupo base. Dicho número indica el tema del texto que cada estudiante debe leer de forma individual. Este estudio es complementado con la ejecución de un laboratorio de historia, donde los alumnos revisan y analizan fuentes históricas físicas y digitales recopiladas por ellos mismos (45 minutos). En los casos de las asignaturas "Ciencias Sociales II" y "Conociendo Chile de los siglos XIX y XX", la mayor parte de la documentación provino de Memoria Chilena (http://www.memoriachilena.gob.cl/602/w3-channel.html) y del Portal de Archivos Españoles (http://pares.mcu.es/); y en el caso de "Didáctica de la Historia, Geografía y Ciencias Sociales I", por no tratarse de una asignatura de Historia propiamente tal, las fuentes de información son de naturaleza curricular que proceden fundamentalmente del sitio de Currículum Nacional (https://www.curriculumnacional.cl/614/w3-channel.html)

Fase IV. Grupos de expertos: Luego del estudio individual, los estudiantes se reúnen con aquellos compañeros que tienen la misma parte, constituyéndose así distintas comisiones de historiadores expertos/as que tienen como fin debatir y analizar el tópico asignado. Este grupo de expertos tiene una duración de 35 minutos. El propósito de esta reunión es doble: por un lado, aprender y profundizar en la temática; y, por otro lado, planificar la manera de transmitir dicho aprendizaje a los demás integrantes del equipo base.

Fase V. Grupos base colaborativos: En esta reunión, los historiadores expertos comunican al grupo los aprendizajes obtenidos de forma autónoma y cooperativa con el grupo de expertos. De igual forma, aprenden todo aquello que les proporcionan sus compañeros sobre el resto del contenido. Esta fase contempla una duración de 35 minutos.

Fase VI. Exposición pública o simulación de clase: En la sesión 2, luego del debate realizado por los grupos base colaborativos, el líder de cada equipo realiza en un lapso de 30 minutos una exposición pública o una simulación de clase sobre las principales ideas analizadas.

Fase VII. Entrega del cuaderno de campo: En la sesión 3, cada estudiante entrega un cuaderno de campo de 6 páginas donde conste una descripción del taller, la fecha de realización, el diseño de un esquema propio que explique la técnica utilizada y la realización de una minuciosa reflexión sobre los desafíos y dificultades del puzle como estrategia de aprendizaje de la Historia.

Figura 1. Fases del puzle

Fase I. Preparación de la experiencia y conformación de los grupos base

Fase II. Introducción al puzle (Sesión 1)

Fase III. Trabajo autónomo (Sesión 1)

Fase IV. Grupo de expertos (Sesión 1)

Fase V. Grupos base colaborativos (Sesión 1)

Fase VI. Exposición pública o simulación de clase (Sesión 2)

Fase VII. Entrega del cuaderno de campo (Sesión 3)

Fuente: Elaboración propia 
REVISTA DE LA ESCUELA DE CIENCIAS DE LA EDUCACIÓN, AÑO 16, NRO. 15, VOL. 2, JULIO A DICIEMRE DE 2020. PÁGINAS 45-57. ISSN 2362-3349 (EN LÍNEA). EL PUZLE COMO TÉCNICA DE APRENDIZAJE COOPERATIVO PARA LA ENSEÑANZA DE LA HISTORIA Y EL DESARROLLO DE HABILIDADES BLANDAS. HUMBERTO ANDRÉS ÁLVAREZ SEPÚLVEDA.

\section{Instrumentos utilizados}

Considerando que la observación participante (Hernández et. al. 2014; Jociles, 2016; Piñeiro y Diz, 2018) es la estrategia principal para la formulación de datos, los instrumentos utilizados para su recogida fueron el registro de asistencia, el cuaderno de campo de los estudiantes y el diario del investigador.

Registro de asistencia: Este insumo de información es indispensable para constatar la concurrencia física de los estudiantes en las clases que contemplaron la preparación y realización del puzle durante las tres sesiones. Cabe destacar que el porcentaje de asistencia es un indicador relevante para evidenciar el grado de responsabilidad y compromiso de los alumnos con la experiencia.

Cuaderno de campo de los estudiantes: Como parte de la actividad, los alumnos elaboraron este instrumento para conocer su percepción sobre la técnica implementada, abarcando aspectos tales como la descripción del taller, la fecha de realización, el diseño de un esquema propio que explique la técnica utilizada y las fortalezas y debilidades que tuvieron en el rompecabezas.

Diario de campo del docente: A lo largo de la experiencia, se ha llevado a cabo un diario de campo donde se han anotado todas aquellas cuestiones relevantes. La observación se planteó desde una perspectiva amplia, intentando obtener una visión general de las sesiones (Pierre y De Sardan, 2019). En cuanto a la estructuración de la observación, cabe destacar que ésta se realizó de manera asistemática, recogiéndose los acontecimientos de forma cronológica, sin utilizar ninguna codificación ni categorías previamente definidas. Una vez analizado el contenido del diario, se construyeron las unidades de observación de manera inductiva, extrayendo las categorías que han permitido su posterior interpretación. De este modo se segmentó la información en unidades de significado, sin perder la visión global (Angrosino, 2012).

\section{Resultados y discusión}

Una vez implementada la experiencia y analizados los datos mediante una triangulación de múltiples fuentes (Delgado y Estepa, 2016), se obtuvieron resultados favorables que permiten constatar la eficacia del puzle para promover el protagonismo del estudiante en su propio proceso de aprendizaje, cuya mejora fue posible gracias a la reflexión que motivó la implementación de esta técnica. siguientes:

Las categorías resultantes tras el análisis de los instrumentos de información consignados son las

Tabla 5. Categorías de análisis con sus respectivos descriptores

\begin{tabular}{|c|c|}
\hline Categorías & Descriptores \\
\hline Aceptación o rechazo de la experiencia & $\begin{array}{l}\text { Actitud favorable por parte del alumnado: ningún } \\
\text { estudiante se niega a ser partícipe de la actividad. } \\
\text { Aceptación de las orientaciones generales } \\
\text { propuestas por el profesor }\end{array}$ \\
\hline Interrelación del equipo & $\begin{array}{l}\text { Resolución consensuada de conflictos, pues } \\
\text { predomina una responsabilidad individual y grupal } \\
\text { efectiva. } \\
\text { No requieren ayuda del profesor. } \\
\text { Se genera una interdependencia positiva entre los } \\
\text { integrantes de cada equipo; no obstante, en algunos } \\
\text { casos, se observan ciertas distracciones y } \\
\text { problemas de afinidad entre los compañeros que } \\
\text { conforman los grupos de expertos. } \\
\text { Se evidencia una fluida interacción cara a cara entre } \\
\text { los miembros de los equipos. } \\
\text { Se observa una reflexión grupal consistente según } \\
\text { los objetivos previstos en la experiencia. }\end{array}$ \\
\hline Implicación del alumnado & $\begin{array}{l}\text { Trabajo autónomo efectivo: evidencian una gran } \\
\text { disposición para participar en la experiencia; } \\
\text { demuestran libertad en la planificación de las tareas; } \\
\text { y entregan orientaciones y ayudas a otros } \\
\text { compañeros. } \\
\text { Cumplen con el cronograma de las sesiones y } \\
\text { demuestran un gran compromiso y participación en } \\
\text { su proceso de aprendizaje. }\end{array}$ \\
\hline
\end{tabular}


REVISTA DE LA ESCUELA DE CIENCIAS DE LA EDUCACIÓN, AÑo 16, NRO. 15, VOL. 2, JULIO A DICIEMRE DE 2020. PÁGINAS 45-57. ISSN 2362-3349 (EN LÍNEA). EL PUZLE COMO TÉCNICA DE APRENDIZAJE COOPERATIVO PARA LA ENSEÑANZA DE LA HISTORIA Y EL DESARROLLO DE HABILIDADES BLANDAS. HUMBERTO ANDRÉS ÁLVAREZ SEPÚLVEDA.

\begin{tabular}{|l|l|ll|l|}
\hline $\begin{array}{l}\text { Valoración de la experiencia por parte del } \\
\text { estudiante }\end{array}$ & $\begin{array}{l}\text { Valoran y reconocen la experiencia como una } \\
\text { instancia enriquecedora para la mejora de } \\
\text { habilidades sociales y la promoción de un } \\
\text { aprendizaje histórico significativo. }\end{array}$ \\
\hline
\end{tabular}

Fuente: Elaboración propia

Con el fin de examinar en mayor profundidad las categorías presentadas, se desarrolla en las próximas líneas un análisis sobre los principales beneficios y dificultades de la experiencia, el cual se centra, fundamentalmente, en la percepción del alumnado.

Como se aprecia en la tabla 5, la implementación del rompecabezas tuvo un impacto positivo en los sujetos participantes, pues permitió evidenciar el desarrollo de habilidades blandas y de aprendizajes significativos relacionados con la Historia y su didáctica.

Dentro de las habilidades blandas, destaca, en primer lugar, el trabajo colaborativo que promovió el puzle en los cursos donde se implementó. En este ámbito, resulta interesante mencionar la interdependencia positiva y la interacción cara a cara entre los integrantes de cada equipo, pues se desenvolvieron eficazmente para conseguir los objetivos trazados en la actividad y lograr el entendimiento entre ellos. Asimismo, dicha fortaleza se observó en el alto grado de coordinación y organización de las tareas que demostró la mayoría de los grupos.

En segundo lugar, la técnica del rompecabezas produjo una mejora efectiva de la comunicación oral en comparación a la constatada en las clases magistrales, lo cual, desde la perspectiva docente, se manifestó en la mayor seguridad y empoderamiento que evidenciaron los estudiantes al momento de exponer los temas consignados frente a sus compañeros. De hecho, el 83\% del alumnado así lo expresó en su cuaderno de campo.

El puzle realizado me permitió reforzar mis capacidades comunicativas y las de mis compañeros, lo que contribuyó a convertir las clases en entornos comunicativos y creativos, en los que participamos, argumentamos y reflexionamos sobre los contenidos estudiados (p.23).

Asimismo, la experiencia contribuyó a fomentar la responsabilidad individual y grupal del equipo de trabajo, pues, como señalan Martínez, Villanueva y Canales (2015), cada estudiante es una pieza del rompecabezas y, por ello, su participación es esencial para el logro de los aprendizajes previstos por el docente.

Esta estrategia no se puede llevar a cabo sin un trabajo conjunto y cohesionado por parte de todo el equipo. Es necesario confiar plenamente en el otro, ya que sólo se tiene conocimiento de una sola parte de la información, por lo que si uno de los integrantes no sabe su parte, todo el grupo flaquea. De esta manera, cada uno puede aprender del otro y así tener la posibilidad de entrelazar habilidades y conocimientos de forma cooperativa (p.17).

Adicionalmente, resulta indispensable destacar la apreciación positiva que hace el alumnado sobre la actividad final del rompecabezas. El 100\% valora la posibilidad de elegir el formato de cierre del taller y el $89 \%$ considera enriquecedora la combinación de los dos métodos (puzle + exposición pública o simulación de clase) por cuanto permite constatar su eficacia conjunta en el desempeño académico del alumnado.

Otra cuestión interesante a destacar es que la experiencia contribuyó a fomentar el compañerismo entre los integrantes de los grupos base del puzle. Esto se vio favorecido porque dichos equipos fueron conformados por afinidad y no por una prescripción docente, lo que facilitó el compromiso grupal, la socialización y la creación de un ambiente propicio para el logro de las metas.

Me sentí muy cómoda realizando esta actividad porque con mi grupo base teníamos gran afinidad y nos complementamos bastante bien al momento de preparar la simulación de clase que debía presentar nuestro líder. Por tal motivo, a mi modo de ver, el rompecabezas es una técnica lúdica y eficaz para que podamos perfeccionar nuestras habilidades interpersonales y de trabajo en equipo (p.34).

En la misma línea, no obstante, el puzle también permitió evidenciar ciertos problemas de afinidad, especialmente en el grupo de expertos, donde los estudiantes tuvieron que debatir con compañeros con los cuales, en algunos casos, no había una buena relación. En este sentido, como señalan Galindo y De la Varga (2016), esta técnica es bastante útil para detectar posibles conflictos en el aula, puesto que la probabilidad de 
REVISTA DE LA ESCUELA DE CIENCIAS DE LA EDUCACIÓN, AÑO 16, NRO. 15, VOL. 2, JULIO A DICIEMRE DE 2020. PÁGINAS 45-57. ISSN 2362-3349 (EN LÍNEA). EL PUZLE COMO TÉCNICA DE APRENDIZAJE COOPERATIVO PARA LA ENSEÑANZA DE LA HISTORIA Y EL DESARROLLO DE HABILIDADES BLANDAS. HUMBERTO ANDRÉS ÁLVAREZ SEPÚLVEDA.

reunir a estudiantes con problemas de socialización es bastante alta bajo la dinámica de funcionamiento del aprendizaje cooperativo.

En el grupo de expertos fue un tanto difícil socializar con mis compañeras, ya que no teníamos gran afinidad entre nosotras. Aun así traté de dar todo de mí parte para que existiera un diálogo, aunque, finalmente, no haya dado resultados positivos (p. 30).

Debido a las ventajas previstas en el ámbito de las habilidades blandas, el $100 \%$ de los estudiantes considera que la experiencia fue altamente significativa y enriquecedora para su crecimiento personal y académico. Esto se aprecia en el alto grado de compromiso y responsabilidad con el que los alumnos realizaron sus respectivos puzles; ello se observa en que el 100\% cumplió con el desarrollo de la actividad.

El compromiso con la experiencia también se manifestó en que el $90 \%$ del alumnado asistió a las clases que contemplaron la organización y preparación del rompecabezas.

También es indispensable resaltar que el 100\% del profesorado en formación inicial justifica la necesidad de replicar y adaptar esta estrategia al contexto de sus futuros alumnos, ya que, según la percepción general de los participantes, no han tenido la posibilidad de utilizarla en la universidad para su propio aprendizaje o de implementarla en las prácticas progresivas que realizan en diversos establecimientos educacionales de la Región del Biobío, en Chile.

Como futura docente sí utilizaría esta técnica en el aula, ya que es muy útil para afianzar las relaciones entre los alumnos y para lograr que comprendan que todos son una pieza clave del aprendizaje cooperativo que se espera alcanzar (p. 26).

Sin embargo, antes de que los docentes en formación inicial puedan implementar el rompecabezas con sus futuros estudiantes, deben considerar algunos problemas que enfrentan las aulas tradicionales. Una de las principales dificultades que presentan las salas de clases es la cantidad de alumnos por curso, cuyo promedio va de 30 a 35 estudiantes. Como destacan Cornejo, Mejías y Sánchez (2017), este problema dificulta la conformación de grupos reducidos, disminuye la motivación y compromiso hacia el aprendizaje, produce una baja constante en la calidad de la enseñanza y limita la creación de un buen clima de aula. Otra posible dificultad para aplicar el puzle es la falta de predisposición que pueden presentar algunos estudiantes para trabajar en equipo.

Además de estas dificultades potenciales, el profesorado en formación también debe prever el nivel escolar, los aprendizajes previos y el contexto educativo de sus futuros alumnos. Al respecto, cabe resaltar que el $100 \%$ de los sujetos participantes están conscientes de estos factores que resultan decisivos de considerar en la implementación del puzle. Por ejemplo, a modo de referencia, el $78 \%$ argumenta que el rompecabezas solo es apto para trabajar con niños de 10 años en delante, porque en los más pequeños puede generar diversos problemas de comprensión lectora debido a su insuficiente madurez cognitiva.

Adicionalmente, coinciden en que desde su rol como futuro profesor deben tener un manejo de aula basado en el liderazgo para disminuir los efectos de las posibles distracciones que pudiesen surgir en el desarrollo de la actividad.

Entre las debilidades que pude constatar en esta técnica, está la alta probabilidad de que el tiempo destinado para el taller puede ser utilizado por los estudiantes para conversar sobre otro tema o bien para hacer desorden. Este riesgo potencial puede aumentar considerablemente si el docente no cuenta con las competencias suficientes para gestionar el clima de aula de un modo efectivo (p.6).

De hecho, cabe destacar que se observaron algunas distracciones en los grupos de trabajo que participaron dentro de la experiencia universitaria. Entre las más recurrentes, se evidenciaron la desatención inicial y el cansancio del alumnado producido por el exceso de carga académica.

Mi gran debilidad fue que, al principio, me costó concentrarme en la lectura porque tenía sueño (cuando aún no nos separaban en grupos). Sin embargo, cuando comenzamos con la dinámica del puzle, debo confesar que la actividad me pareció entretenida y significativa para aprender de una forma diferente los contenidos de la asignatura (p. 14).

En cuanto al desarrollo de aprendizajes relacionados con la Historia y su didáctica, es importante mencionar que la experiencia también ha producido importantes avances en este ámbito.

Una primera cuestión a destacar es que el laboratorio histórico realizado dentro del puzle fue una instancia idónea en la fase de trabajo autónomo y en el grupo de expertos (donde los estudiantes simularon ser historiadores), ya que, siguiendo a Reisman (2012) y a Boutonnet (2013), ayudó al alumnado a concebir la 
REVISTA DE LA ESCUELA DE CIENCIAS DE LA EDUCACIÓN, AÑO 16, NRO. 15, VOL. 2, JULIO A DICIEMRE DE 2020. PÁGINAS 45-57. ISSN 2362-3349 (EN LÍNEA). EL PUZLE COMO TÉCNICA DE APRENDIZAJE COOPERATIVO PARA LA ENSEÑANZA DE LA HISTORIA Y EL DESARROLLO DE HABILIDADES BLANDAS. HUMBERTO ANDRÉS ÁLVAREZ SEPÚLVEDA.

Historia como un conocimiento que se construye a partir de una serie de operaciones metodológicas que tienen como propósito hacer frente a un problema específico con una interpretación cuidadosa de las fuentes de información.

Además del avance epistémico mencionado, el laboratorio histórico también ofreció la oportunidad de simular métodos de investigación propios del área de la Historia, cuyo modelo fue aprovechado por el profesorado en formación inicial para desarrollar su pensamiento histórico y su respectivo proceso de alfabetización. Así, el rompecabezas permitió a los estudiantes conocer la historia más próxima; comprender el pasado y su relevancia en el presente; promover la autonomía personal en la propia reconstrucción de la Historia; y caracterizar el conocimiento histórico como discutible y problematizador.

De este modo, los alumnos no se limitaron a aprender pasivamente un conocimiento ya elaborado, sino que fueron los auténticos protagonistas de su propio aprendizaje, como es lógico, siguiendo el camino trazado por el profesor y contando con toda la información, materiales y recursos preparados para desarrollar el puzle. Así, siguiendo a Salazar, Orellana, Muñoz y Bellati (2017), el estudiante puede llegar a «descubrir» explicaciones, desde las más simples a las relativamente complejas sobre hechos, fenómenos y acontecimientos pasados, deducir regularidades, secuencias o, incluso, elaborar explicaciones históricas.

El laboratorio de historia implementado en el rompecabezas me motivó a «pensar históricamente» las problemáticas analizadas y a familiarizarme con las destrezas cognitivas asociadas al manejo de fuentes. Estos conocimientos, sin duda, son fundamentales para enfrentarse a la invasión de información y noticias de los medios y redes sociales, frecuentemente incompletas, sesgadas o directamente manipuladas (p.9).

En la línea prevista, y siguiendo los planteamientos didácticos del pensamiento histórico, no se trata de que los docentes en formación se instruyan como historiadores, sino que sean capaces de transferir las competencias históricas al área pedagógica y de valorar las diferentes interpretaciones que se hacen del pasado para describir a las sociedades actuales (Schmidt, 2016).

Por añadidura, cabe notar que el rompecabezas permitió ampliar y profundizar los contenidos que fueron tratados en cada curso, pues tuvieron la posibilidad de analizar, juzgar e interpretar los hechos y sus consecuencias desde diferentes puntos de vista.

La técnica del puzle, especialmente el desarrollo del grupo de expertos, fue muy relevante para comprender desde diferentes perspectivas algunas de las motivaciones que impulsaron al conquistador español a venir a Chile y para poder dimensionar la relevancia histórica de las expediciones de Almagro y Valdivia en el presente. No obstante, también me permitió constatar que el proceso de conquista está dominado por un discurso histórico androcéntrico que relega la participación de las mujeres a un segundo plano en dicho periodo histórico (p. 19).

A partir de la experiencia didáctica, pude comprender de forma significativa las múltiples causas internas y externas que influyeron en el proceso de Independencia de Chile y sobre la forma en cómo éstas se interrelacionaron para configurar esta etapa de la historia de nuestro país. Esto, sin duda, no lo hubiese podido lograr con un modo de enseñanza tradicional, ya que bajo este paradigma el docente solo se hubiera limitado a transmitir contenidos memorísticos sesgados por una lógica eurocéntrica y relacionados con grandes personajes y conflictos bélicos de la Independencia (p. 25).

Considerando como base las últimas percepciones citadas, es importante señalar que el $61 \%$ de la muestra señala en su cuaderno de campo que la experiencia didáctica les ayudó a evidenciar el androcentrismo y la proyección de aprendizajes de primer orden en la historia oficial que se concibe en el currículum escolar chileno. En este contexto, este porcentaje del alumnado coincide en la necesidad de reivindicar el papel de la mujer como un sujeto histórico que ha aportado de forma relevante al desarrollo de la humanidad. Este mismo segmento también plantea la urgencia de analizar y desarrollar en el estudiante los aprendizajes de segundo orden que contempla el pensamiento histórico (tabla 1).

La necesidad de repensar la historia oficial, a través del uso de metodologías activas, es cada vez más latente debido a que la investigación reciente ha constatado la invisibilización de las mujeres y la ausencia de la promoción del pensamiento histórico en la enseñanza de la especialidad. La exclusión de tales cuestiones es un hecho reconocible tanto en las programaciones curriculares como en las prácticas del profesorado (Ramallo, 2014; Ortega y Pagès, 2018; Ortega, 2018).

Otro aspecto interesante de resaltar es que esta experiencia fue fundamental para que los profesores en formación inicial obtuvieran los aprendizajes teóricos necesarios para implementar el puzle en forma correcta, lo cual es un insumo clave si es que tienen que replicar esta técnica con sus futuros estudiantes o en otras asignaturas de su carrera. Por tal motivo, el saber hacer del rompecabezas, y muy especialmente del 
REVISTA DE LA ESCUELA DE CIENCIAS DE LA EDUCACIÓN, AÑo 16, NRO. 15, VOL. 2, JULIO A DICIEMRE DE 2020. PÁGINAS 45-57. ISSN 2362-3349 (EN LÍNEA). EL PUZLE COMO TÉCNICA DE APRENDIZAJE COOPERATIVO PARA LA ENSEÑANZA DE LA HISTORIA Y EL DESARROLLO DE HABILIDADES BLANDAS. HUMBERTO ANDRÉS ÁLVAREZ SEPÚLVEDA.

laboratorio histórico incluido, fue la fase mejor lograda y más valorada por el alumnado, ya que, aparte de estudiarla como un concepto, tuvieron la posibilidad de aprenderla en la praxis.

Debido a esto último, la totalidad de los estudiantes considera que el puzle es una estrategia mucho más útil y significativa en su proceso formativo que el desarrollo de evaluaciones tradicionales, centradas en la repetición y memorización de hitos universales o historias nacionales que narran la hazaña de grandes personajes.

Los resultados positivos logrados con el rompecabezas implementado son coherentes a los obtenidos en otras experiencias similares realizadas en distintas carreras y niveles educativos de la enseñanza obligatoria (Mayorga y Madrid, 2012; Llorent y Varo, 2013; Galindo y De la Varga, 2016). Esto evidencia que esta técnica puede generar un impacto favorable en diversos contextos, pues contribuye no sólo a la adquisición de competencias y contenidos, sino también a la formación integral del individuo.

\section{Conclusiones}

La experiencia didáctica, que surgió a raíz de una preocupación personal, permitió obtener resultados congruentes que demuestran la eficacia del rompecabezas para generar un aprendizaje significativo en los estudiantes, pues, según la perspectiva docente y la de ellos mismos, lograron potenciar sus habilidades blandas y mejorar su comprensión sobre la Historia y su didáctica.

Para continuar con este itinerario de tendencia constructivista dentro de la enseñanza de la Historia, se espera introducir cambios metodológicos que permitan promover un aprendizaje cooperativo entre los alumnos y que, a su vez, facilite el logro de los objetivos trazados para las distintas actividades curriculares.

Estos resultados favorables ratifican la necesidad de reorientar la enseñanza universitaria de la Historia hacia enfoques didácticos más constructivistas y cercanos al mundo profesional, puesto que las universidades se ven cada vez más inmersas en la creciente globalización de las actividades humanas y en la masificación de las TIC.

Sin embargo, no hay que olvidar que el docente debe prever posibles retos y dificultades en la implementación del puzle, tales como: la imposibilidad del estudiante de acudir regularmente a clase; la actitud pasiva del alumnado; el escepticismo sobre sus ventajas; la sobrecarga de trabajo; y el miedo a las habilidades sociales.

\section{Referencias bibliográficas}

Angrosino, M. (2012). Etnografía y observación participante en investigación cualitativa. Madrid: Morata. Aronson, E. y Blaney, N. (1978). The jigsaw classroom. Oxford: Sage.

Barca, I. (2011). Narrativas e consciência histórica dos jovens. En: Enseñanza de las Ciencias Sociales, 10, pp. 2228.

Barton, K. (2010). Investigación sobre las ideas de los estudiantes acerca de la Historia. En: Enseñanza de las Ciencias Sociales, 9, pp. 97-114.

Biberhofer, P.; Lintner, C., Bernhardt, J. y Rieckmann, M. (2018). Facilitating work performance of sustainabilitydriven entrepreneurs through higher education: The relevance of competencies, values, worldviews and opportunities. En: The International Journal of Entrepreneurship and Innovation, 20(1), pp. 21-38. DOI: https://doi.org/10.1177/1465750318755881

Boutonnet, V. (2013). Les ressources didactiques: typologie d'usages en lien avec la méthode historique et l'intervention éducative d'enseignants d'histoire au secondaire. Montréal: Université de Montréal.

Cornejo, E.; Mejías, L. y Sánchez, C. (2017). El enfoque por tareas y el manejo de aula en las clases de inglés de enseñanza media. En: Revista Contextos, Estudios de Humanidades y Ciencias Sociales, 38, 1-19.

Delgado, E. y Estepa, J. (2016). Ciudadanía y memoria histórica en la enseñanza de la historia: análisis de la metodología didáctica en un estudio de caso en ESO. En: Revista de Investigación Educativa, 34(2), pp. $521-534$.

Díaz, A. y Luna, A. (2014). Metodología de la investigación educativa. México: Díaz de Santos.

Escudero, T. (2016). La investigación evaluativa en el siglo XXI: Un instrumento para el desarrollo educativo y social cada vez más relevante. En: Relieve, 22(1), pp. 1-21.

Galindo, F. y De la Varga, J. (2016). El método puzle como técnica para el aprendizaje cooperativo de los mapas estratégicos: Una experiencia en la asignatura "Administración de Organizaciones". En: Repositorio Institucional de la Universidad de Málaga (RIUMA), pp. 1-10.

García, J. (2019). Desarrollo de competencias para el siglo XXI. Miami: Humboldt International University.

Gómez, M. (2006). Introducción a la metodología de la investigación científica. Córdoba: Brujas.

Gómez, C., López, R., Miralles, P. y Prats, J. (2017). Enseñanza de la historia y competencias educativas. Barcelona: Graó.

Gómez, C.; Rodríguez, R. y Mirete, A. (2018). Percepción de la enseñanza de la historia y concepciones epistemológicas. Una investigación con futuros maestros. En: Revista Complutense de Educación, 29(1), pp. 237-250. 
REVISTA DE LA ESCUELA DE CIENCIAS DE LA EdUCACIÓN, AÑo 16, NRO. 15, VOL. 2, JULIO A DICIEMRE DE 2020. PÁGINAS 45-57. ISSN 2362-3349 (EN LÍNEA). EL PUZLE COMO TÉCNICA DE APRENDIZAJE COOPERATIVO PARA LA ENSEÑANZA DE LA HISTORIA Y EL DESARROLLO DE HABILIDADES BLANDAS. HUMBERTO ANDRÉS ÁLVAREZ SEPÚLVEDA.

González, P. y Núñez, L. (2012). El puzle de la historia: Una experiencia innovadora en Historia del Mundo Contemporáneo basada en el aprendizaje cooperativo. En: Edetania, 42, pp. 129-143.

Hernández, R.; Fernández, C. y Baptista P. (2014). Metodología de la investigación. México: McGraw-Hill Interamericana.

Jociles, M. (2016). La observación participante: ¿consiste en hablar con "informantes"? En: Quaderns-e de I'Institut Català d'Antropologia, 21(1), pp. 113-124.

Johnson, D.; Johnson, R. y Johnson, E. (1999). El aprendizaje cooperativo en el aula. Buenos Aires: Paidós.

Levstik, L. S. y Barton, K. C. (2011). Doing history. Investigating with children in elementary and middle schools. Nueva York: Routledge.

Llorent, V. y Varo, J. (2013). Innovaciones didácticas para fomentar el aprendizaje y la cohesión social en el aula. La técnica puzle con equipos sabios. En: Innovación educativa, 23, 179-196.

Martínez, R.; Villanueva, M. y Canales, E. (2015). Aprendizaje corporativo: Una alternativa para mejorar la instrumentación didáctica en la Educación Superior Tecnológica. Madrid: Díaz de Santos.

Mayorga, J. y Madrid, D. (2012). La técnica del puzle como estrategia de aprendizaje cooperativo para la mejora del rendimiento académico. En: Publicaciones, 42, pp. 1-26.

Melero, N. (2012). El paradigma crítico y los aportes de la investigación acción participativa en la transformación social: Un análisis desde las Ciencias Sociales. En: Cuestiones Pedagógicas, 21, pp. 339-355.

Miralles, P. y Monteagudo, J. (2019). Métodos, instrumentos y procedimientos para conocer cómo se evalúan las competencias históricas. En: Educar em Revista, 35(74), pp.127-144.

Ortega, D. (2018). Las mujeres en la historia enseñada: Resultados de un programa docente en formación inicial del profesorado. En: Enseñanza de las Ciencias Sociales, 17, pp. 13-21.

Ortega, D. y Pagès, J. (2018). Género y formación del profesorado: análisis de las guías docentes del área de Didáctica de las Ciencias Sociales. En: Contextos Educativos. Revista de Educación, 21, pp. 53-66.

Pierre, J. y De Sardan, O. (2019). El rigor de lo cualitativo: las obligaciones empíricas de la interpretación socioantropológica. Madrid: Centro de Investigaciones Sociológicas (CIS).

Pinos, M. (2019). Con corazón y cerebro: Net learning: aprendizaje basado en la neurociencia, la emoción y el pensamiento. Madrid: Penguin Random House.

Prats, J. (2011). Didáctica de la Geografía y la Historia. Barcelona: Editorial Graó.

Prats, J. (2017). Retos y dificultades para la enseñanza de la historia. En P. Sanz, J. Molero y D. Rodríguez (Eds). La historia en el aula. Innovación docente y enseñanza de la historia en la educación secundaria (15-32). Lleida: Milenio.

Ramallo, F. (2014). Enseñanzas de la historia y lecturas descoloniales: entrecruzamientos hacia los saberes de otros mundos posibles. En: Revista Entramados - Educación y Sociedad, 1(1), pp. 43-59.

Reisman, A. (2012). Reading like a historian: A document-based history curriculum intervention in urban high schools. En: Cognition and Instruction, 30(1), pp. 86-112.

Rivera O., P. y Mondaca R., C. (2018). El aporte de la enseñanza de la historia reciente en Chile: Disensos y consensos desde la transición política al siglo XXI. En: Estudios Pedagógicos, 39(1), pp. 393-401. DOI:10.4067/S0718-07052013000100023

Rodríguez, A.; Souto, J. y Arroyo. M. (2019). Improving teaching capacity to increase student achievement: The key role of communication competences in Higher Education. En: Studies in Educational Evaluation, 60, pp. 205-213. DOI: https://doi.org/10.1016/j.stueduc.2018.10.002

Rumbo, B. y Gómez, T. (2019). Estudio del modelo de aprendizaje por competencias para la empleabilidad en la universidad. En: Revista de Pedagogía, 39(105), pp. 241-259.

Sáiz, J. y Domínguez, J. (2017). Aprender sobre la historia: competencias metodológicas en educación secundaria. En C. Gómez, R. López, P. Miralles y J. Prats (Eds), Enseñanza de la historia y competencias educativas (23-47). Barcelona: Graó.

Salazar, R.; Orellana, C., Muñoz, C. y Bellati, I. (2017). El aula como laboratorio histórico: la guerra civil española. En C. Gómez, R. López, P. Miralles y J. Prats (Eds), Enseñanza de la historia y competencias educativas (110126). Barcelona: Graó.

Schmidt, M. (2016). Globalización y la política de formación del profesor de Historia en Brasil. En: Perspectiva Educacional. Formación de Profesores, 55(1), pp. 38-50. DOI: 10.4151/07189729-Vol.55-Iss.1-Art.357

Silva, J. y Maturana, D. (2017). Una propuesta de modelo para introducir metodologías activas en educación superior. En: Innovación Educativa, 17(73), pp. 117-131.

Simons, H. (2011). Estudio de caso: Teoría y práctica. Madrid: Morata. 